\title{
GENETIC CONTROLS OF RECOMBINATION \\ I. THE RECOMBINATION-2 GENE OF NEUROSPORA CRASSA
}

\author{
BRIAN R. SMITH \\ Department of Genetics, Australian National University, \\ Conberro, A.C.T., Australia
}

Received 7.i.66

\section{INTRODUCTION}

STUDIEs of allelic recombination have, for the most part, been directed towards mapping the sites of genetic differences within genes. Mapping of these sites depends primarily on the frequencies of prototrophs formed amongst the progeny of crosses between pairs of auxotrophic alleles made in the repulsion phase. It is assumed that the frequency of prototrophs is a measure of the physical distance between the sites at which two auxotrophs differ from one another. Thus any pair of auxotrophic alleles would show a frequency which is the sum or the difference of the frequencies shown by crosses of these alleles to a third auxotroph. Although, in many cases it has been possible to construct maps using this additivity rule in practice any substantial body of data shows inconsistencies so that often the frequencies are only very poorly additive.

Commonly fine structure mapping is not solely dependent on prototroph frequencies, but also on the distribution of flanking markers amongst the prototrophs produced in allelic crosses. In a cross of the general type $A m^{1} B \times a m^{2} b$ where $A$ and $a$ are the proximal and $B$ and $b$ the distal flanking markers, the prototrophs, $m^{+}$fall into four classes. These are the two parental combinations $A B$ and $a b$ and the two recombinants $A b$ and $a B$. In Neurospora no one recombinant class vastly exceeds any other class and all four occur with substantial frequencies. However, some asymmetries are observed. The recombinant classes are often unequal in size. The parental classes are often unequal in size, and the directions of inequality of the parental and recombinant classes are correlated. It has often been assumed that the inequalities are due to "normal " crossing over and that the high frequencies of parental and the second recombinant classes are due to the simultaneous occurrence of two or three cross overs indicating a high degree of "negative interference". However, high negative interference has been invoked merely as a description of the factor which is necessary to fit the observations to the cross over theory of recombination.

Relationships used to determine the order of sites of mutation are best understood if it is assumed that recombination does occur by a process analogous to crossing over. Three criteria have been considered (Jessop and Catcheside, 1965. Smith, I965). 
I. If the recombinant classes are compared, the order will be $A m^{1} m^{2} B$ if $a B$ is in the majority and will be $A m^{2} m^{1} B$ if $A b$ is in the majority (see fig. I).

2. If the proximal flanking alleles only are considered, the order will be $A m^{1} m^{2}$ if $a$ is in the majority and will be $A m^{2} m^{1}$ if $A$ is in the majority. In other words, the more frequent proximal flanking allele identifies the more distal of the $m$ allele differences since they entered the cross together.

3. If the distal flanking alleles are considered, the order will be $m^{1} m^{2} B$ if $B$ is in the majority and will be $m^{2} m^{1} B$ if $b$ is in the majority. That is, the more frequent distal flanking allele identifies the more proximal of the $m$ allele differences since they entered the cross together.
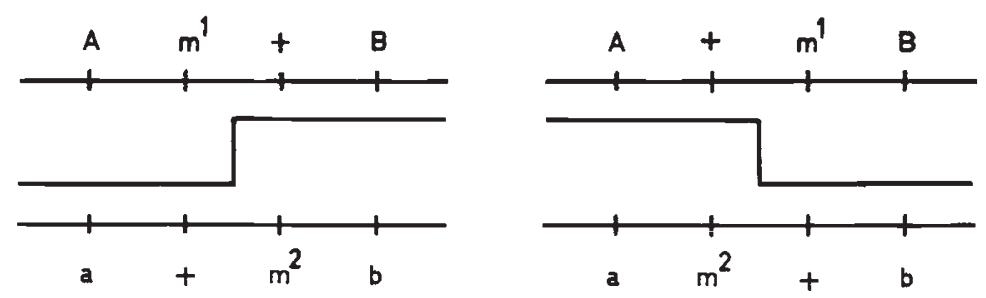

FIg. 1.-Diagrams to show the derivation of the criterion for ordering the sites of difference $m^{1}$ and $m^{2}$ based on the distribution of flanking markers $A$ and $B$ amongst prototrophic recombinants $m^{+}$.

In a few cases all the criteria lead to the derivation of a consistent order for a series of alleles and this order agrees for the most part with the order based on prototroph frequencies (Jessop and Catcheside, 1965; Smith, 1965). However, the remarkable feature of recombination data from various organisms is the great variability which exists not only between species, but also between genes in the same species and even between the same pairs of alleles (Catcheside, unpubl.).

Variation is not confined to allelic recombination but has long been recognised in crosses between nonallelic genes. Variation in this respect takes the form of differences in the frequency of recombination between the same linked genes when stocks of different origin are used. This variation is so marked in some species that accurate linkage maps can only be constructed using data from three or four point crosses.

In the past interest has been centred on chromosome mapping, fine structure mapping and on the mechanism of "conversion ". In these respects most of the variability observed has seemed more of a nuisance than a source of information about mechanisms (Catcheside, Jessop and Smith, 1964). It has now been recognised that close study of all these variations will provide information essential to an understanding of the process of recombination.

The work reported here is part of a detailed study of variations encountered in the course of mapping the fine structure of the his-5 gene in Neurospora crassa (Smith, 1965). It was observed in this earlier 
work that the frequency of prototroph formation in crosses between the same pair of his -5 alleles could vary by a factor of about two depending on the stocks used. In the few cases where recombination between the flanking markers in the general population of these crosses was determined related frequency changes were recorded. In addition, the distribution of flanking markers amongst allelic prototrophic recombinants often differed significantly although the variations were not sufficient to reverse the order of sites of difference deduced using the three marker criteria. Since it was thought that all these variations are genetically determined the present investigation was designed to show the ways in which these differences are inherited and the relationships between them.

\section{MATERIALS AND METHODS}

\section{(a) Organisms}

The two histidine-5 mutants, $\mathrm{K}_{5} 12$ and $\mathrm{K}_{553}$, were isolated (Catcheside, 1960) from the wild type Emerson a by filtration enrichment, following irradiation with ultra-violet light. The pyrimidine-3 mutant allele used was 1298 originally received from Dr G. W. Beadle. A leucine-2 mutant 37501, kindly donated by Dr D. D. Perkins, was successively outcrossed to the Emerson A wild type three times before use, only a single isolate, of mating type A, from the third cross being used in the present study. Apart from these four mutants the only other stocks which have entered the pedigree are the wild types Emerson a and Emerson A. Pyr-3, his-5 and leu-2 are linked in the right arm of chromosome IV, pyr-3 being about one map unit proximal to his-5 and leu-2 about nine map units distal to his-5 (Smith, 1962).

\section{(b) Media}

All stocks were grown and kept on slopes of Vogel's medium (Vogel, 1955), suitably supplemented. Crosses were made, using mixed inocula, on slopes of Westergaard's medium (Westergaard and Mitchell, 1947) containing folded strips of filter paper. Cultures were tested for biochemical requirements on Vogel's medium in which 0.5 per cent. sorbose and 0.1 per cent. sucrose replaced the usual 2 per cent. sucrose. Supplements of growth factors were added to the media where necessary, to give the following concentrations; for histidine mutants, $500 \mathrm{mg} . / \mathrm{l}$. of L-histidine mono-hydrochloride, for pyrimidine mutants $500 \mathrm{mg}$./l. of uracil and for leucine mutants $600 \mathrm{mg}$./1. of DL-leucine.

\section{(c) Crosses}

Each cross for analysis of histidine prototroph frequency and recombination between flanking marker genes was made using mixed inocula in about eight tubes of crossing medium and ascospores from the different tubes were pooled. The ascospores were collected in sterile water, filtered through muslin, and after removal of excess water by decantation, suspended in $0 \cdot 1$ per cent. agar. An estimate of the number of spores per $\mathrm{ml}$. of suspension was obtained by counting the number of spores in $0.01 \mathrm{ml}$. drops of suspension on slides on which the area covered by the coverslip was divided into sixty-four squares.

The frequency of recombination between flanking markers in the general population of the crosses was estimated using a selective method. After germination of the ascospore suspension by heat treatment at $56^{\circ} \mathrm{C}$. for 60 minutes, volumes of spore suspension containing approximately 2,000 spores were pipetted into tubes containing $2.5 \mathrm{ml}$. volumes of 0.8 per cent. agar supplemented with histidine and kept molten 
at $45^{\circ} \mathrm{C}$. The contents of the tubes were quickly mixed and evenly spread over plates of Vogel's medium. For each cross six of the plates were supplemented with histidine and four with histidine, uracil and leucine. After incubation at $25^{\circ} \mathrm{C}$. for 18 hours the plates were inspected under a binocular microscope and each spore scored for growth. Estimates of the viability of the spores were obtained from the four plates containing histidine, uracil and leucine. From the plates containing only histidine estimates of the frequency of the recombinant class that is prototrophic with respect to pyr-3 and leu-2 were obtained, only this class of spores being able to grow on the medium. The frequency of prototrophic recombinants, corrected for the viability of the spores, which invariably exceeded 90 per cent., was doubled to give the frequency of recombination between the marker genes.

Recombination between the his-5 alleles in the various crosses was measured by estimating the frequency of histidine prototroph formation amongst the ascospores. For this, suspensions of ascospores, heat treated as before, were added to 0.7 per cent. agar supplemented with uracil and leucine kept molten at $45^{\circ} \mathrm{C}$. to give a concentration of about 25,000 ascospores per $\mathrm{ml}$. A sample of this suspension was then further diluted in molten 0.7 per cent. agar containing uracil and leucine to give a final concentration of between 20 and 35 spores per $\mathrm{ml}$. Samples $(3 \mathrm{ml}$.) of this suspension were then pipetted on to Petri plates containing Vogel's medium in which the normal 2 per cent. sucrose was replaced by 1 per cent. sorbose, 0.025 per cent. glucose and 0.025 per cent. fructose and which was also supplemented with histidine, uracil and leucine. The remainder of the concentrated spore suspension, containing about 25 , 000 spores per ml., was pipetted in $3 \mathrm{ml}$. lots on to plates of similar medium but supplemented only with uracil and leucine. The plates were incubated at $25^{\circ} \mathrm{C}$. and scored for growth under a binocular microscope after 48 hours. The plates were re-examined using the naked eye on the succeeding 2 days after which time no further colonies appeared. From the plates supplemented with histidine, uracil and leucine an accurate estimate of the total number of viable spores in the original spore suspension was obtained. Histidine prototrophs appearing on the plates containing uracil and leucine were transferred to slopes of Vogel's medium containing uracil and leucine and subsequently tested for their flanking marker requirements.

\section{RESULTS}

It was found that differences in the rates of recombination between the his- 5 alleles were not related to the frequencies of recombination between the flanking markers in the general population of the crosses. Consequently, these two aspects of the data will be considered separately. For convenience the genotype of every stock used in the study is shown in the pedigree in table $I$.

\section{(i) Analysis of recombination between the flanking marker genes in crosses of the form pyr-3 his-5 leu- $2^{+} \times$pyr $-3^{+}$his -5 leu- 2}

The analysis had best begin with a pair of stocks of the distal marker leu-2. Two isolates of $\mathrm{K}_{553}$ leu-2*, both of mating type A, were obtained from the cross +leu-2 A $\times \mathrm{K}_{553}+\mathrm{a}$. These two isolates are designated $\mathrm{K}_{553}$ leu-2 ( I) A and $\mathrm{K}_{553}$ leu-2 (3) A. Each was crossed to pyr-3 $\mathrm{K}_{5} \mathrm{I}_{2}$ (3) a, a stock isolated from the cross $p y r-3+\mathrm{A} \times+\mathrm{K}_{5} \mathrm{I} 2 \mathrm{a}$,

* In writing the constitution, only the allele numbers of the his-5 mutants will be given Strictly the constitution here should be written his-5 $\mathrm{K} 553$ leu-237501, but since only one mutant allele of leu-2 and pyr-3 have been employed, the simpler form of the genotype is unambiguous. 


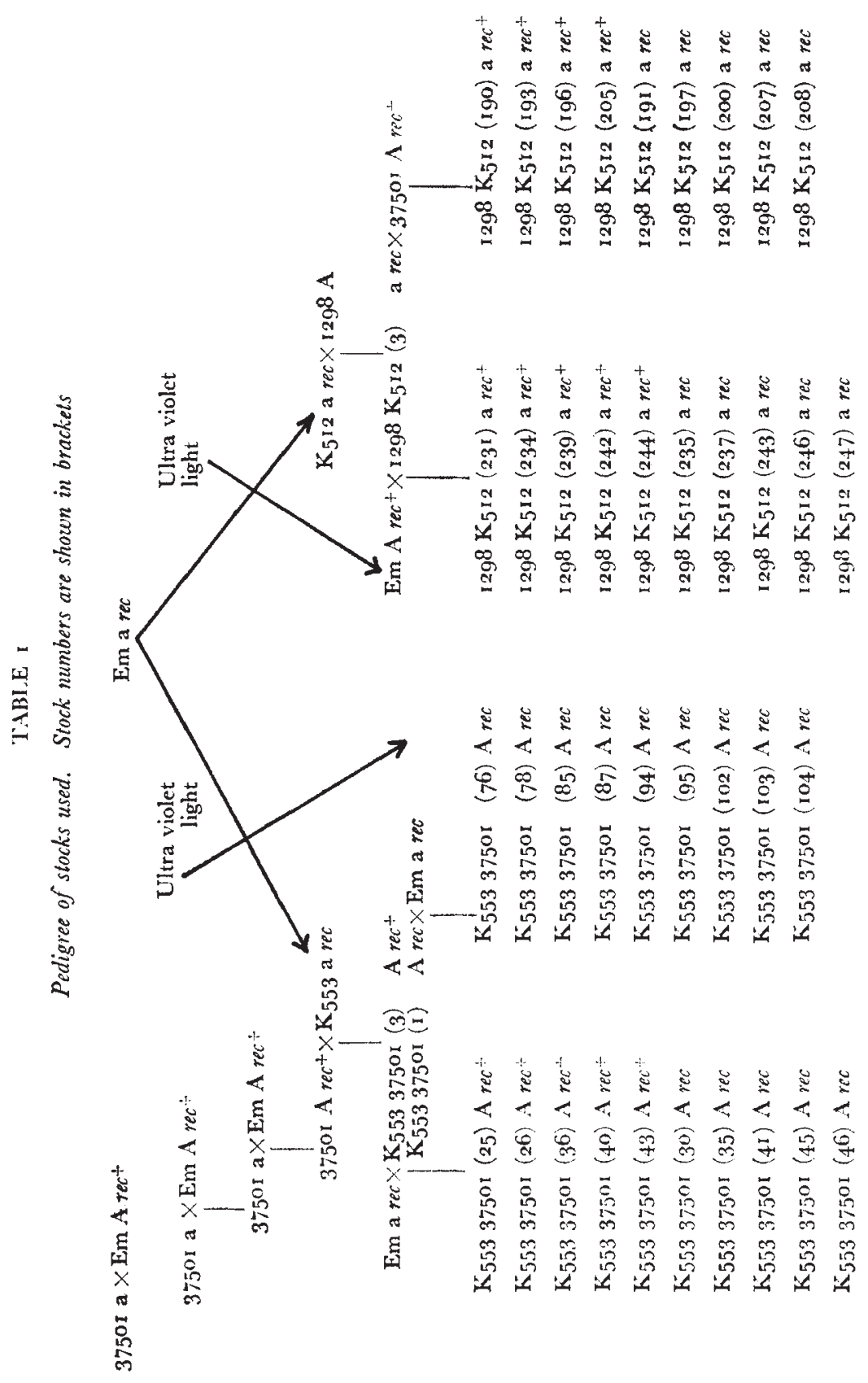


and the frequency of recombination between $p y r-3$ and leu-2 in the progeny determined by the selective method. The results (table 2 entries $\mathrm{I}$ and 2) show that $\mathrm{K}_{553}$ leu-2 ( $\mathrm{I}$ ) A exhibits a recombination frequency of $2 \mathrm{I}^{\prime} 5$ per cent. with pyr-3 $\mathrm{K}_{512}$ (3) a whereas $\mathrm{K}_{553}$ leu-2 (3) A shows a frequency of 9.95 per cent., a little under half that of the first cross. Both the $\mathrm{K}_{553}$ leu-2 stocks were then out-crossed to $\mathrm{Em}$ a and $\mathrm{K}_{553}$ leu-2 isolates of mating type $\mathrm{A}$ recovered. These isolates were crossed with pyr-3 $\mathrm{K}_{5} \mathrm{I}_{2}$ (3) a and estimates of the recombination frequencies obtained. Ten isolates from the cross $\mathrm{K}_{553}$ leu-2 (3) $\mathrm{A} \times \mathrm{Em}$ a were tested (entries 3 and 4 in table 2). Five of these gave frequencies of about ro per cent. and five of about 20 per cent. Of nine isolates from the cross $\mathrm{K}_{553}$ leu-2 (I) $\mathrm{A} \times \mathrm{Em}$ a tested with pyr-3 $\mathrm{K}_{5}$ I2 (3) a all gave values around 20 per cent. (entry 5 in table 2 ).

It is clear at this stage that the differences in the frequencies of recombination between pyr-3 and leu-2 are produced by a single genetic difference segregating, as far as one can tell, independently of leu-2 and mating type. If the locus concerned is designated recombination-2, the allele for low frequency may be symbolised as $\mathrm{rec}^{+}$and for high frequency as rec. In these terms, $\mathrm{K}_{553}$ leu-2 (I) A is rec and $\mathrm{K}_{553}$ leu-2 (3) A is rec ${ }^{+}$. When the rec stock was crossed with $\mathrm{Em}$ a all the progeny tested with pyr-3 $\mathrm{K}_{5} \mathrm{I} 2$ (3) a were rec, whereas the progeny of the cross $\mathrm{Em} \mathrm{a} \times \mathrm{rec}^{+}$yielded five $\mathrm{rec}$ and five $\mathrm{rec}^{+}$stocks. This shows that $\mathrm{Em}$ a is itself rec, and since $\mathrm{K}_{553}$ and $\mathrm{K}_{5 \mathrm{I}} 2$ were isolated from $\mathrm{Em}$ a they too are rec. The rec ${ }^{+}$allele present in the stock $\mathrm{K}_{553}$ leu-2 (3) A must also be present in the original leu-2 mutant as it was the only other stock to enter the pedigree.

At this point it was not known whether the low or high frequency allele is dominant as the constitution of $p y r-3 \mathrm{~K}_{5} \mathrm{I} 2$ (3) a with respect to the recombination-2 gene was unknown. In an effort to determine the constitution of pyr-3 $\mathrm{K}_{512}$ (3) a it was crossed to the leu-2 mutant $375^{\circ} \mathrm{I} \mathrm{A}$, which as we have seen must be $\mathrm{rec}^{+}$and further pyr-3 $\mathrm{K}_{5} \mathrm{I} 2 \mathrm{a}$ stocks reisolated. When crossed to the rec stock $\mathrm{K}_{553}$ leu-2 (I) A, four of these isolates gave frequencies of recombination of about ro per cent. and five gave frequencies around 20 per cent. (entries 6 and 7 of table 2). This segregation indicates that pyr-3 $\mathrm{K}_{5} \mathrm{I} 2$ (3) a is rec. The same nine isolates, four of them $\mathrm{rec}^{+}$and five rec, when crossed with the rec ${ }^{+}$ stock $\mathrm{K}_{553}$ leu-2 (3) A all yielded recombination frequencies of about Io per cent. (entries 8 and 9 in table 2). These results indicate that the $\mathrm{rec}^{+}$allele is dominant to the rec allele. That is, if one parent bears the $\mathrm{rec}^{+}$allele then the lower frequency of recombination will result regardless of which allele is carried by the other parent.

The leu-2 mutant $375^{\circ}$ I A rec ${ }^{+}$used in this study was, as already stated, reisolated after three successive crosses to Emerson A so it was thought likely that the $\mathrm{rec}^{+}$allele is also carried by $\mathrm{Em} \mathrm{A}$. In order to test this possibility and to confirm that rect is dominant to rec, pyr-3 $\mathrm{K}_{5} \mathrm{I} 2$ (3) a rec was crossed to $\mathrm{Em} \mathrm{A}$ and ten pyr-3 $\mathrm{K}_{5} \mathrm{I} 2$ a cultures extracted and crossed to $\mathrm{K}_{553}$ leu-2 (I) A rec. Five of the crosses with 


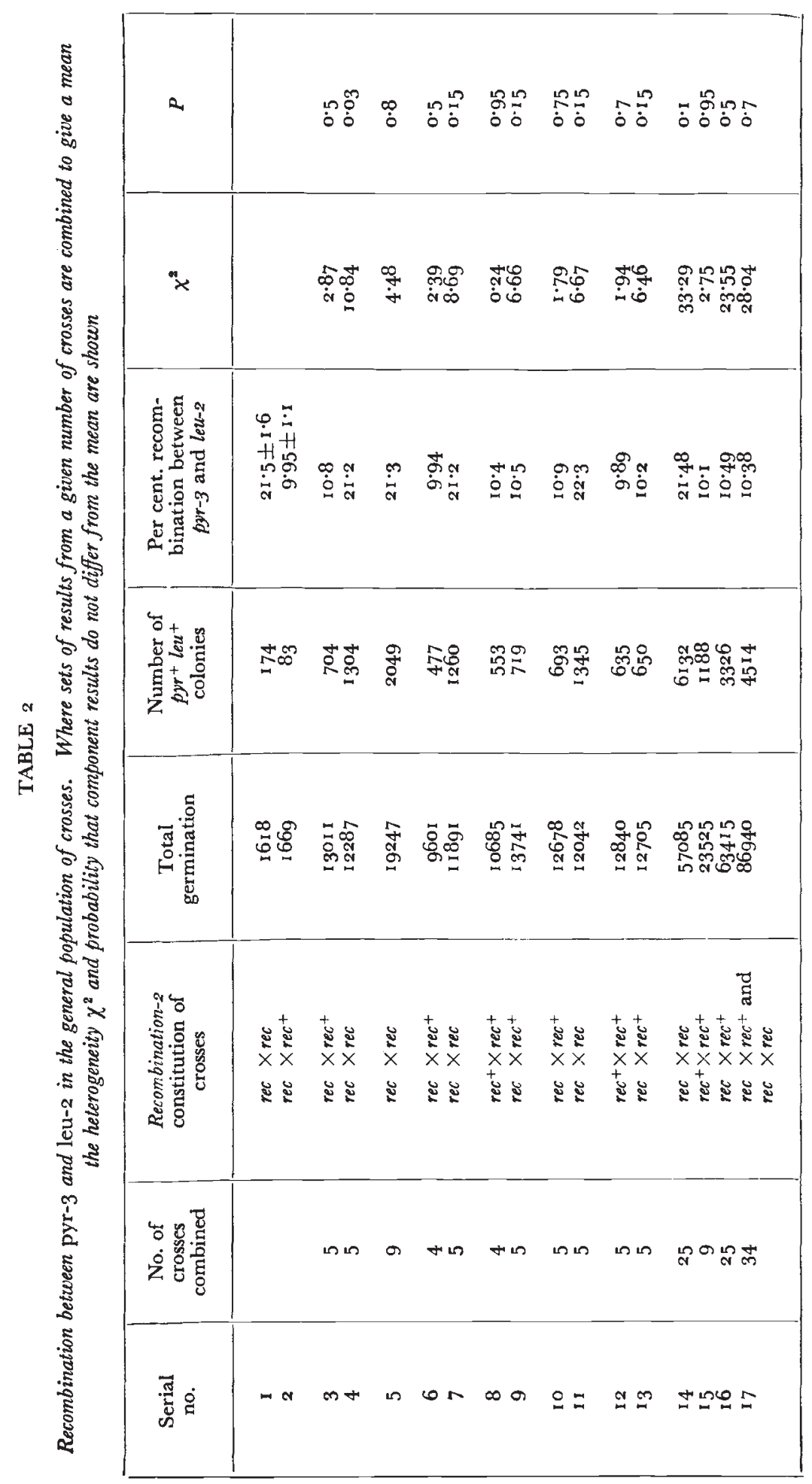


the rec stock $\mathrm{K}_{553}$ leu-2 (I) A gave about 10 per cent. recombination and five about 20 per cent. (entries 10 and $I \mathrm{I}$ in table 2), indicating that $\mathrm{Em} \mathrm{A}$ is in fact rec ${ }^{+}$. All ten of the pyr-3 $\mathrm{K}_{512}$ a isolates gave around 10 per cent. recombination when crossed with $\mathrm{K}_{553}$ leu-2 (3) A rec ${ }^{+}$, (entries 12 and 13 of table 2) a finding that confirms dominance of rec ${ }^{+}$over rec.

Of the fifty-nine crosses examined twenty-five are $\mathrm{rec}^{+} \times \mathrm{rec}$, twentyfive are rec $\times$ rec and nine are $\mathrm{rec}^{+} \times \mathrm{rec}^{+}$. Considering the $\mathrm{rec} \times \mathrm{rec}$ crosses, the actual frequencies of recombination observed vary from $18 \cdot 2 \pm 1 \cdot 1$ to $24 \cdot 4 \pm 1 \cdot 4$. The weighted mean value for the recombination in all the rec $\times$ rec crosses is $21 \cdot 48$ per cent. (entry 14 in table 2). Though the frequency of recombination in the rec $\times$ rec crosses is in every case considerably higher than the highest frequency of the $\mathrm{rec} \times \mathrm{rec}^{+}$or $\mathrm{rec}^{+} \times \mathrm{rec}^{+}$crosses it is apparent that they are less homogeneous than either $\mathrm{rec} \times \mathrm{rec}^{+}$or $\mathrm{rec}^{+} \times \mathrm{rec}^{+}$crosses. It has not been possible to determine why these minor differences occur although it is thought that genetically determined modifiers are operating and that the effect of these is somewhat masked in crosses bearing the dominant rec-2 allele.

Considering next the $\mathrm{rec} \times \mathrm{rec}^{+}$crosses, the frequencies of recombination vary from $8 \cdot 45 \pm 0 \cdot 82$ to $12 \cdot 0 \pm 0 \cdot 98$. The extreme recombination values amongst the $\mathrm{rec}^{+} \times \mathrm{rec}^{+}$crosses were $9.39 \pm 0.86$ per cent. and $10.9 \pm 0.97$ per cent. The average value for recombination in all the rec $\times \mathrm{rec}^{+}$crosses is 10.49 per cent. (entry 16 in table 2 ). This compares with an average of ${ }_{10} \cdot \mathrm{I}^{\mathrm{P}}$ per cent. in the $\mathrm{rec}^{+} \times \mathrm{rec}^{+}$crosses (entry ${ }_{15}$ in table 2). The difference between these values is not significant (entry 17 in table 2). Comparing the average values of recombination of the $\mathrm{rec}^{+} \times \mathrm{rec}$ and $\mathrm{rec}^{+} \times \mathrm{rec}^{+}$crosses, $10 \cdot 3^{8}$ per cent., with the average value for the $r e c \times r e c$ crosses, $21 \cdot 4^{8}$ per cent., the latter is $2 \cdot 1$ times the former.

All the recombination values considered so far were obtained by the selective method in which it is assumed that the two parental classes occur with equal frequency and also that the recombinant classes are equal, as is normally found in crosses between linked loci. To confirm that this is also true in the present work, three of the crosses were selected for complete analyses, in which random spores were grown and subsequently tested for their marker requirements. Between them the crosses provide all the combinations of the rec-2 alleles. It is apparent from the results (table 3 ) that there are no significant differences between the two parental classes of spores nor between the recombinant classes. Considering all three crosses there is some indication of a deficiency of the leu-2 mutant classes probably attributable to a slightly reduced viability of this mutant. The recombination values observed in these experiments are almost identical to the values for the same crosses analysed by the selective method (table 3 ).

At present there is no difinite indication of the location of the rec-2 gene. The data already presented do however provide some information in this respect. Progeny from three crosses that were of one parental 


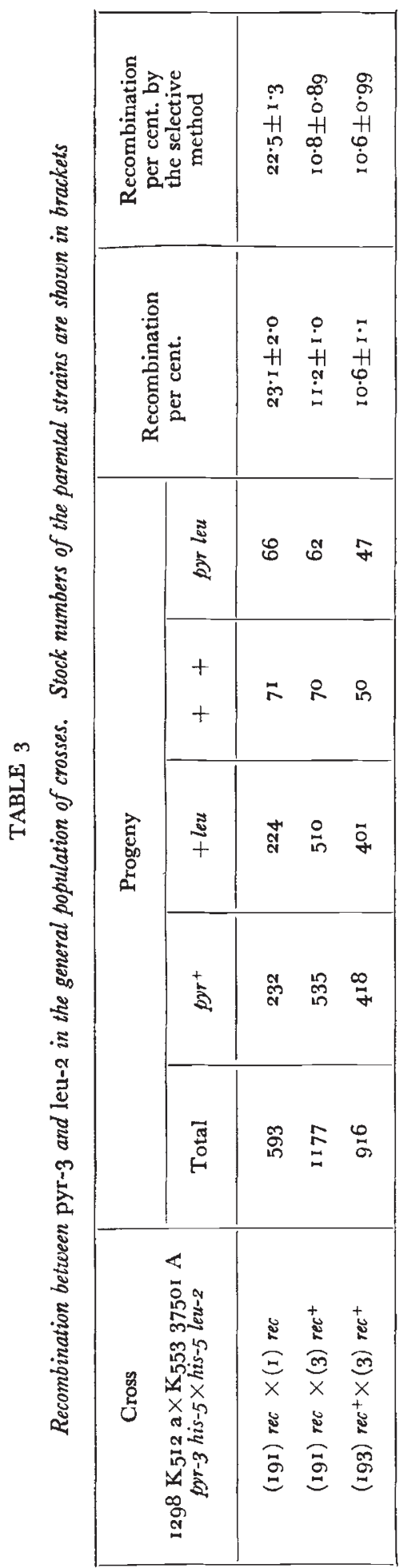


type with respect to his-5 and mating type have been isolated and their constitution with respect to the rec-2 differences determined. In these crosses, pyr-3 $\mathrm{K}_{5}$ I2* (3) a rec $\times 375$ o I $\mathrm{A} \mathrm{rec}^{+}$, pyr-3 $\mathrm{K}_{5 \mathrm{I}} 2^{*}$ (3) a rec $\times \mathrm{Em}$ A rec ${ }^{+}$and $\mathrm{K}_{553}$ leu-2* (3) A rec $\times \mathrm{Em}$ a rec, the parental class isolated in each case is indicated by an asterisk. Of the 29 isolates from these crosses, I5 were parental for the rec-2 gene and I4 were recombinant. There is no evidence from these rather limited data for linkage of $r e c-2$ with mating type on group I or his-5 on group IV.

\section{(ii) Allelic recombination}

Recombination between the his-5 alleles $\mathrm{K}_{512}$ and $\mathrm{K}_{553}$ was also studied in all the crosses which were previously used to determine recombination between $p y r-3$ and leu-2. Crosses were analysed for their frequency of formation of histidine prototrophs and the prototrophs were then classified for the four combinations of flanking markers, two parental pyr + and +leu, and two recombinant, + + and pyr leu. The data for all the crosses are summarised in table 4.

(a) Prototroph frequencies. Amongst the fifty-nine crosses in table 4, the frequency of prototroph formation varies between 5.28 per $\mathrm{IO}^{5}$ ascospores and 12.7 per $10^{5}$ spores. Between these extremes the values are evenly distributed. These frequencies show no relationship with the rec-2 constitution of the crosses as had been expected (Smith, 1965). Indeed it has not been possible to demonstrate any relation between the ancestry of the parents of the crosses and the resulting frequencies of prototroph formation. In view of the number of sampling errors inherent in the experimental method it is not practicable to determine to what extent the extremes of frequency observed in the data are significant. These extremes are about the same as those observed in previous data (Smith, 1965), where the highest frequency for a given pair of alleles was little more than twice the lowest.

(b) Distribution of flanking markers. The data upon the distribution of flanking markers are shown in table 4. In earlier data (Smith, I 965; Jessop and Catcheside, r 965 ) it was found possible to ascertain with some confidence which of any pair of allelic differences was proximal to the other by examining the distribution of flanking markers amongst the resulting prototrophic recombinants. The three criteria used for ordering the sites of mutation have already been stated (see introduction).

Applying the first criterion to the present data the recombinant class of prototrophs in the majority in all the crosses is the pyr-3 leu-2 double mutant class, indicating that $\mathrm{K}_{553}$ is proximal to $\mathrm{K}_{5} \mathrm{r} 2$. Considering the proximal flanking marker distribution, criterion II, the pyr-3 mutant class is in the majority in all crosses, again indicating that $\mathrm{K}_{553}$ is proximal to $\mathrm{K}_{512}$. The distal marker distribution, criterion III, is much nearer to equality than is the proximal marker distribution. Even so, all, except three crosses, have the leucine class 

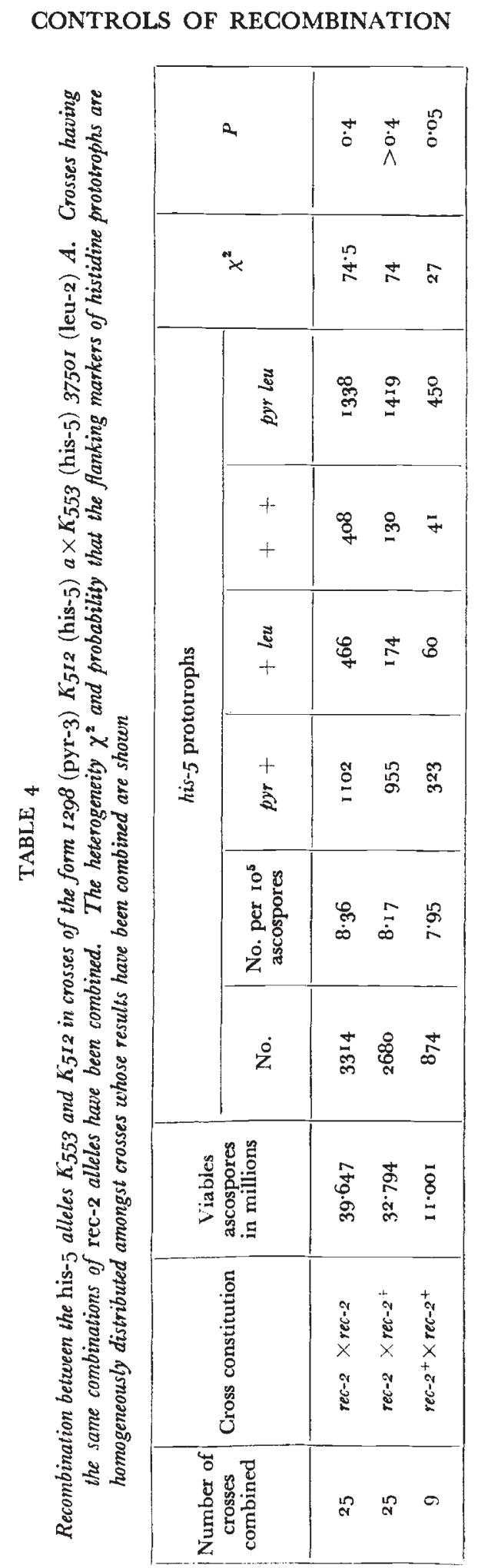
in the majority, once more indicating that $\mathrm{K}_{553}$ is proximal to $\mathrm{K}_{512}$. In the three exceptional crosses the differences are not significant.

Closer examination of the distribution of the flanking markers amongst the histidine prototrophs reveals a number of interesting points. In the $25 \mathrm{rec} \times \mathrm{rec}^{+}$crosses (table 4 ) the distribution of the flanking markers is homogeneous. The heterogeneity $\chi^{2}$, for 72 degrees of freedom, is 74 and $\mathbf{P}>0.4$. Similarly the distribution of the markers amongst the prototrophs in the $9 \mathrm{rec}^{+} \times \mathrm{rec}^{+}$crosses (table 4) is also considered homogeneous, the $\chi^{2}$ for 24 degrees of freedom being 27 and $\mathbf{P}$ is 0.05 . It should be appreciated that in many cases the number of prototrophs in a particular class is small giving inflated contributions to $\chi^{2}$ so that the probability is greater than the figures suggest. The

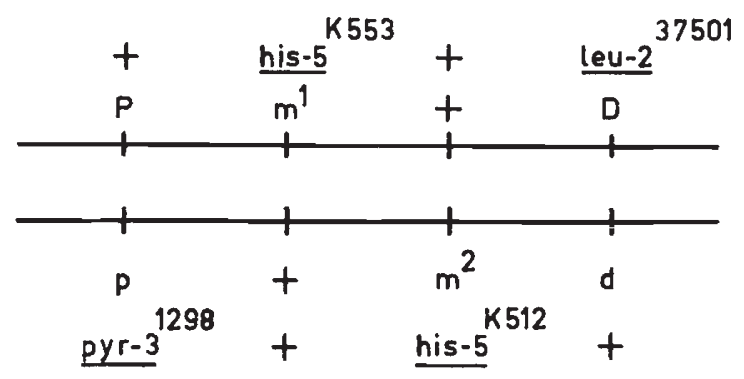

Fig. 2-Formalised representation of crosses. For full explanation see text.

distribution in the $\mathrm{rec}^{+} \times \mathrm{rec}^{+}$crosses is almost identical to that in the $\mathrm{rec} \times \mathrm{rec}^{+}$crosses.

The distribution of markers amongst prototrophs of the $25 \mathrm{rec} \times \mathrm{rec}$ crosses (table 4 ) is homogeneous, $\chi^{2}$ for 72 degrees of freedom being 74.5 and $\mathrm{P}$ just $<0.4$. The distribution in these $r e c \times r e c$ crosses differs significantly from that of the $\mathrm{rec} \times \mathrm{rec}^{+}$and $\mathrm{rec}^{+} \times \mathrm{rec}^{+}$crosses. Before considering this difference in detail it is convenient to formalise the constution of the crosses (fig 2), by referring to proximal marker differences as $P$ and $p$, the distal marker differences as $D$ and $d$ and the auxotrophic allelic differences as $m^{1}$ and $m^{2}, m^{1}$ being proximal to $m^{2}$ (Gatcheside, unpubl.).

We are now in a position to examine the distribution of each flanking marker separately. This is most easily displayed by means of square root charts in which $\sqrt{D}$ is plotted against $\sqrt{d}$, or $\sqrt{P}$ against $\sqrt{p}$. Taking the distal marker first (fig. 3 ), there is very little difference in the distribution although what difference there is may be real. It is not very convincing, but the spread of the points suggests that two populations are involved.

Turning to the distribution of the proximal flanking marker (fig. 4), there are very significant differences. All the $r e c \times r e c$ crosses have a much larger proportion of the $p y r-3^{+}$or $P$ prototrophs than do the 
$\mathrm{rec} \times \mathrm{rec}^{+}$or $\mathrm{rec}^{+} \times \mathrm{rec}^{+}$crosses. If $x$ is the chance that a prototroph is $P$ then the value of $x$ for $\mathrm{rec} \times \mathrm{rec}^{+}$and $\mathrm{rec}^{+} \times \mathrm{rec}^{+}$crosses is $\mathrm{O} \cdot \mathrm{I}$ I 5 whereas the value for $r e c \times r e c$ crosses is $0 \cdot 26$, this being $2 \cdot 3$ times greater than in the crosses containing the dominant $\mathrm{rec}^{+}$allele.

If $y$ is the chance that a prototroph is $D$ the expectations for each class of prototroph may be calculated on the assumption that the

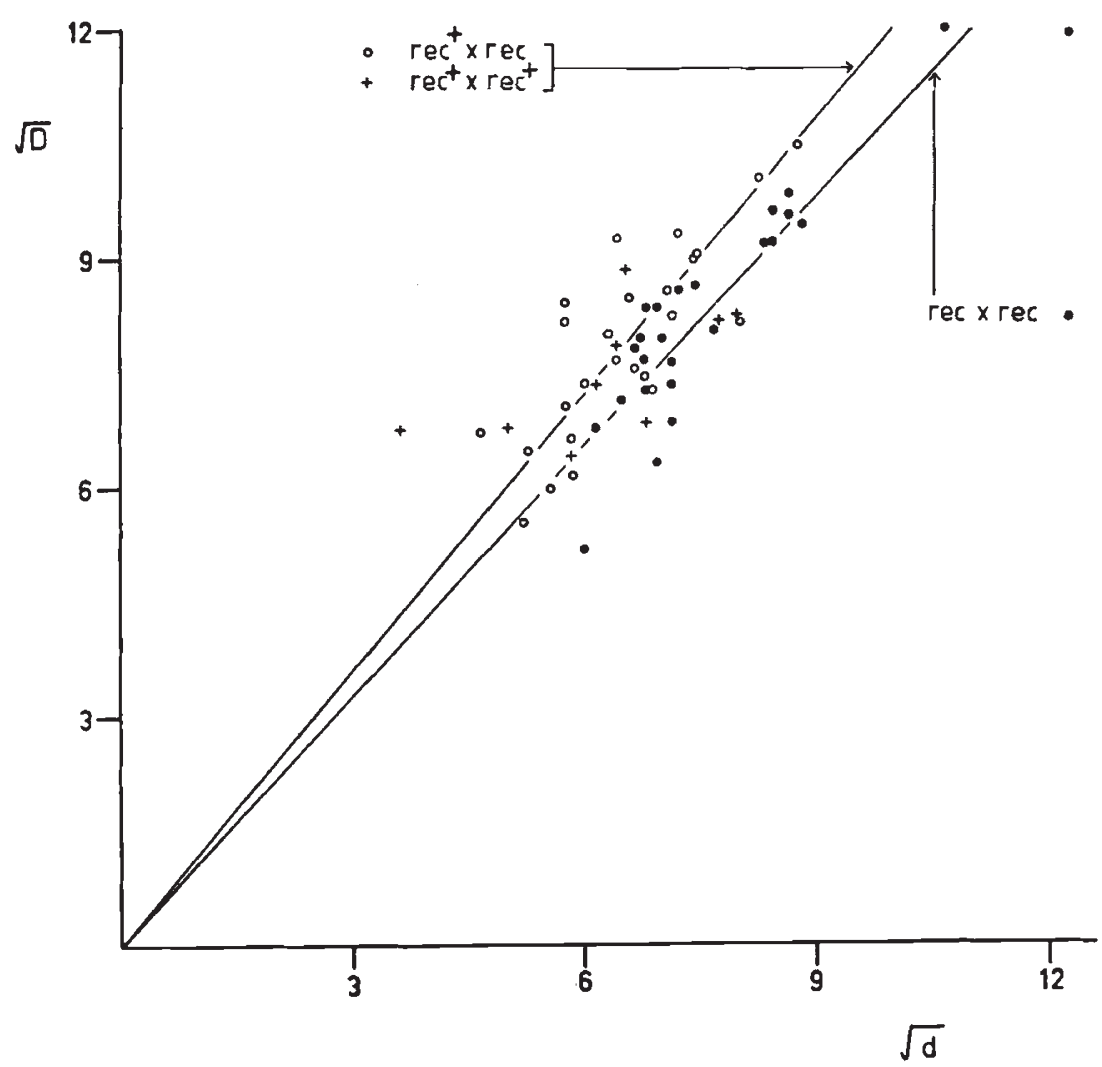

Frg. 3.-Showing the effects of rec-2 differences on the distribution of the distal flanking marker amongst prototrophic allelic recombinants. Each point represents the result from a single cross of indicated $r e c-2$ constitution. For full explanation see text.

probabilities $x$ and $y$ are independent (Catcheside, unpubl.). Algebraically the probability for each class is as follows:

$\begin{array}{ll}\text { Class } & \text { Probability } \\ p d & (\mathrm{I}-x)(\mathrm{I}-y) \\ P D & x y \\ P d & x(\mathrm{I}-y) \\ p D & (\mathrm{I}-x) y\end{array}$

Table 5 shows the observed totals and the expected numbers in each class of prototroph for the three types of crosses and also for the 
$r e c \times r e c^{+}$and $r e c^{+} \times r e c^{+}$crosses combined. The heterogeneity $\chi^{2}$ and probability that the expected numbers do not differ from the observed numbers are shown and in each case the observations do not differ significantly from the calculated. It is fairly certain therefore that the two flanking markers are associated independently with the prototrophs to form the four combinations.

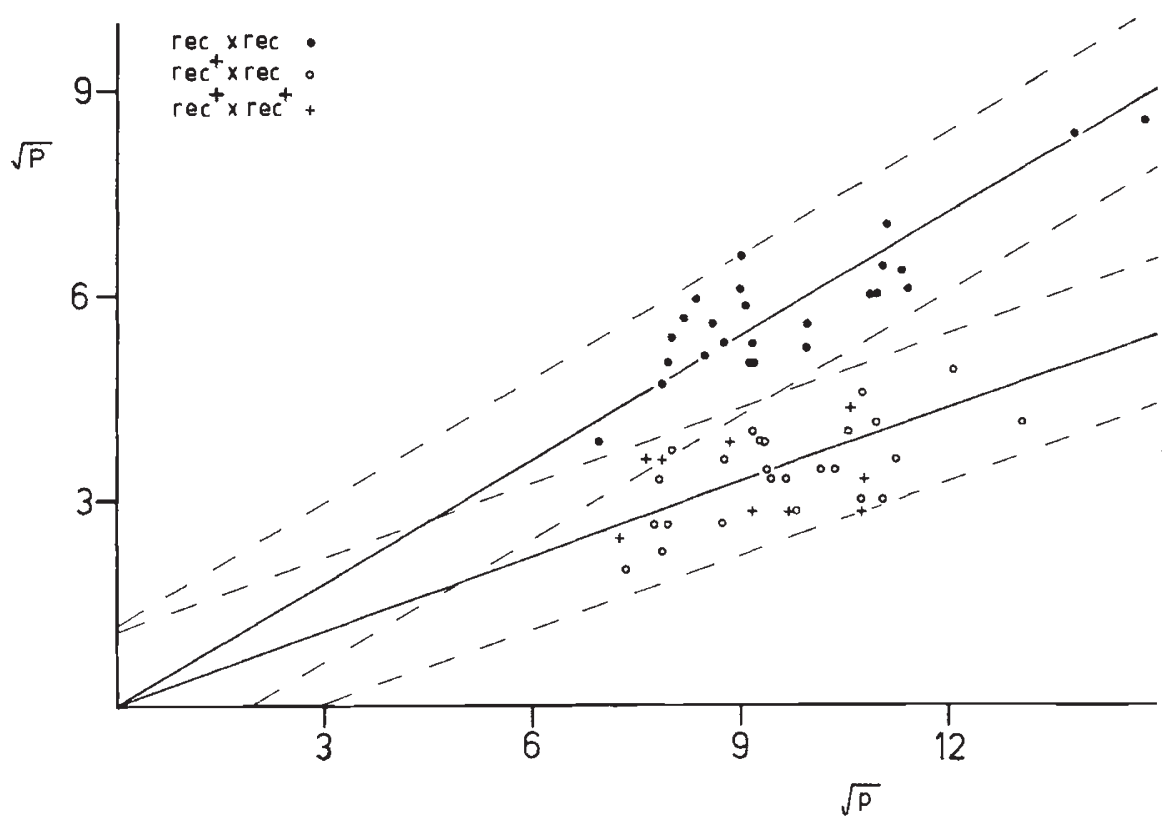

Fig. 4.--Showing the effects of rec-2 differences on the distribution of the proximal flanking marker amongst prototrophic allelic recombinants. Each point represents the result from a single cross of indicated rec-2 constitution. Broken lines indicate the 95 per cent. confidence limits to the two weighted means shown as continuous lines.

The main effects of the recombination-2 gene on recombination are best seen when recombination between the flanking markers in the general population of the crosses is plotted against the value of $x$ for the crosses (fig. 5). The presence of the dominant rec ${ }^{+}$allele in a cross, in either homozygous or heterozygous condition, invariably leads to a reduction in the rate of recombination between flanking markers and to a reduction in the value of $x$.

\section{DISCUSSION}

Theories of recombination have been proposed that take account of the molecular structure of deoxyribonucleic acid and of its time of replication with respect to the time of genetic recombination at meiosis (Holliday, I962, I964; Whitehouse, I 963; Hastings and Whitehouse, I964; Whitehouse and Hastings, 1965). These proposals include, variously, separation over a short distance of the two chains of a DNA 
molecule, the breakage of one or both of these separated chains, the formation of segments of DNA of hybrid origin, the excision of mispaired bases or segments of a DNA chain, and the reconstruction of segments of the DNA chain using information in the other chain. Many of these events would involve chemical reactions. It is characteristic of organisms that such reactions are catalysed by enzymes and that each enzyme is structurally specified by a gene which may be controlled either directly or indirectly by other genes. It is in these terms that the rec-2 differences must be interpreted.

TABLE 5

Showing the observed and expected numbers of prototrophs in each marker class for crosses of particular rec-2 recombinations

\begin{tabular}{|c|c|c|c|c|c|c|c|}
\hline \multicolumn{2}{|c|}{ Crosses } & $\begin{array}{c}p y r+ \\
p d\end{array}$ & $\begin{array}{l}+ \text { leu } \\
P D\end{array}$ & ${ }_{P d}^{+}$ & $\begin{array}{c}\text { pyr leu } \\
p D \\
p D\end{array}$ & $\chi^{2}$ & $P$ \\
\hline$r e c \times r e c$ & $\begin{array}{l}\text { Observed } \\
\text { Expected }\end{array}$ & $\begin{array}{l}\text { I I } 102 \\
\text { I I I I I } \cdot 8\end{array}$ & $\begin{array}{l}466 \\
475 \cdot 9\end{array}$ & $\begin{array}{l}408 \\
398 \cdot 9\end{array}$ & $\begin{array}{l}\text { I } 338 \\
\text { I } 328 \cdot 3\end{array}$ & 0.614 & 0.4 \\
\hline $\mathrm{rec}^{+} \times \mathrm{rec}$ & $\begin{array}{l}\text { Observed } \\
\text { Expected }\end{array}$ & $\begin{array}{l}955 \\
9^{6 I} \cdot 2\end{array}$ & $\begin{array}{l}176 \\
182 \cdot I\end{array}$ & $\begin{array}{l}130 \\
123.9\end{array}$ & $\begin{array}{l}\text { I } 419 \\
\text { I } 412 \cdot 8\end{array}$ & $0.57^{2}$ & 0.4 \\
\hline $\mathrm{rec}^{+} \times \mathrm{rec}^{+}$ & $\begin{array}{l}\text { Observed } \\
\text { Expected }\end{array}$ & $\begin{array}{l}323 \\
321 \cdot 9\end{array}$ & $\begin{array}{l}60 \\
58 \cdot 9\end{array}$ & $\begin{array}{l}4 \mathrm{I} \\
4^{2} \cdot \mathrm{I}\end{array}$ & $\begin{array}{l}45^{\circ} \\
45^{1} \cdot \mathrm{I}\end{array}$ & 0.0557 & 0.8 \\
\hline $\left.\begin{array}{l}\mathrm{rec}^{+} \times \mathrm{rec}^{+} \\
\mathrm{rec}^{+} \times \mathrm{rec}^{+}\end{array}\right\}$ & $\begin{array}{l}\text { Observed } \\
\text { Expected }\end{array}$ & $\begin{array}{l}\text { I } 278 \\
\text { I } 283\end{array}$ & $\begin{array}{l}23^{6} \\
241 \cdot 1\end{array}$ & $\begin{array}{l}\text { I } 71 \\
165.9\end{array}$ & $\begin{array}{l}\text { I } 869 \\
\text { I } 864^{\cdot} \cdot \text { I }\end{array}$ & $0 \cdot 297$ & 0.7 \\
\hline
\end{tabular}

In the first place, it is apparent that rec-2 is fully recessive to $r e c-2^{+}$, that is a single dose of $r e c-2^{+}$is as effective as two doses. It is an axiom that dominance implies that the gene product is metabolically active or that the mutation is in an operator gene. Hence there are two general modes of action of rec-2 ${ }^{+}$, either it determines the structure of an enzyme which catalyses one of the events which lead to genetic recombination or else it determines the structure of a regulatory substance. In practice, it would be difficult to distinguish between these possibilities.

The two main effects of the rec-2 ${ }^{+}$allele are, first a reduction in frequency of recombination between non allelic genes to about half the frequency observed in crosses homozygous for the rec-2 allele and second a reduction in the frequency of allelic prototrophic recombinants bearing the less frequent proximal flanking marker to a little under half the frequency in crosses homozygous for rec-2. It is reasonable to assume at this stage that these effects are general, affecting all regions of each chromosome rather than restricted to the particular system studied. This assumption is being tested as fully as possible in current experiments. 
It might be thought that the change in frequency of the less frequent proximal flanking marker class of allelic prototrophic recombinants from 0.26 in crosses homozygous for rec-2 to 0. I I $_{5}$ in crosses bearing $r e c-2^{+}$in one or both parents could be accounted for by the increase in non allelic recombination between the proximal marker pyr-3 and his-5.

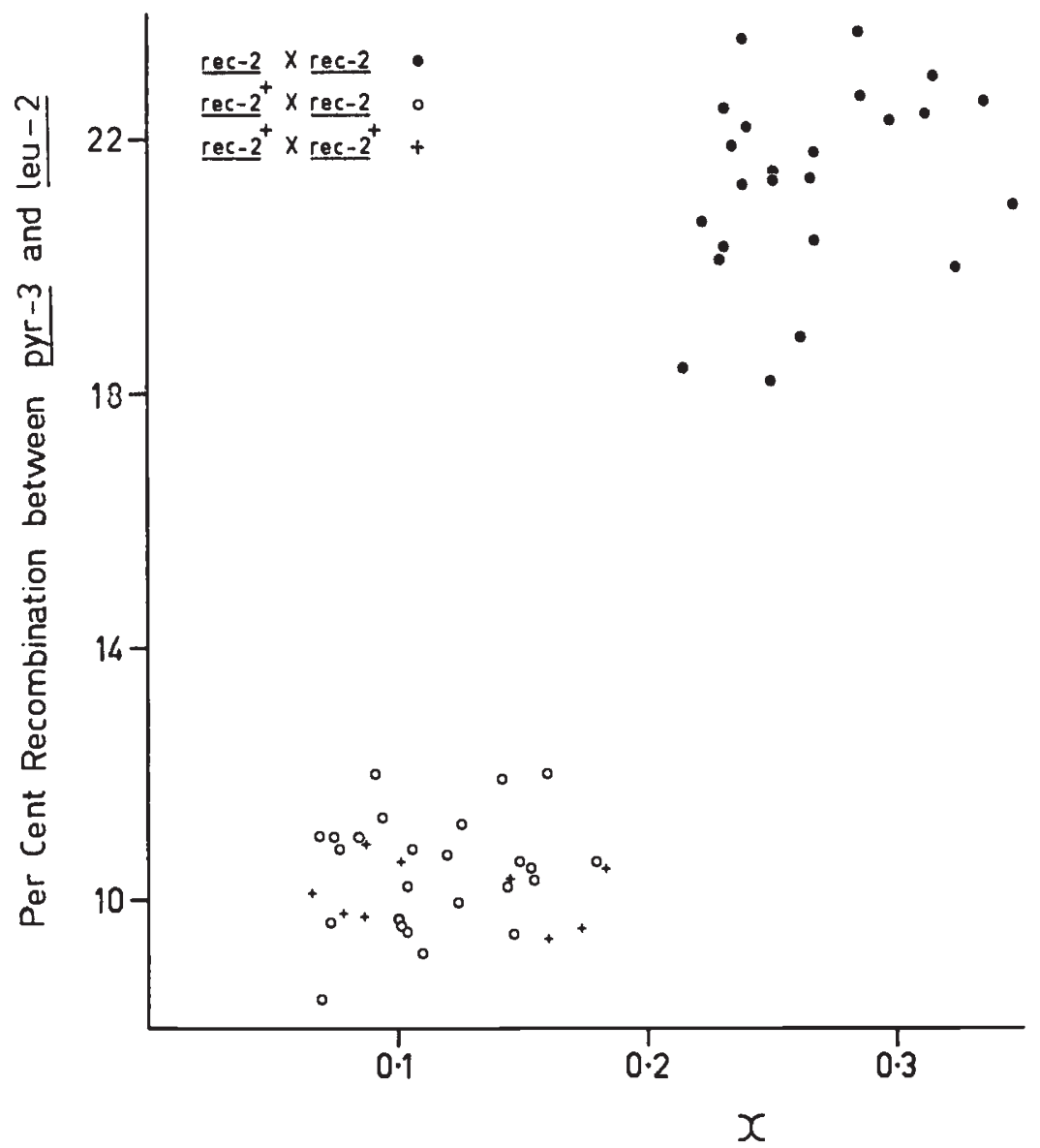

FIG. 5.-Showing the main effects of recombination-2 on allelic and non allelic recombination. For each cross, of indicated rec-2 constitution, the per cent. recombination between pyr-3 and leu-2 is plotted against the value of $x$. For full explanation see text.

However, to get such a change would require an increase in recombination between pyr-3 and his-5 from I per cent. in crosses bearing rec-2 + in one or both parents to I 9.8 per cent. in crosses homozygous for rec-2. Such an increase would be $\mathrm{I} \cdot 7$ times greater than the total increase in recombination frequency observed between the two flanking markers pyr-3 and leu-2. Thus the rec-2 gene appears to control both allelic and non allelic recombination, a phenomenon which adds considerable weight to the theory that allelic and non allelic recombination occurs by essentially the same mechanism. 
The very different effects of the rec-2 genetic differences on allelic and non allelic recombination remain unexplained but they must reflect in some way the action of the genetic differences over very small and very large segments of the chromosome respectively.

It is already apparent that adequate interpretation of the action of individual genes which control recombination will not be possible until more such genes are identified and their mode of action determined. Of equal, if not greater, value will be the study of the interaction of recombination genes.

In Neurospora only one other genetic difference controlling recombination has been analysed in any detail. This is the recombination gene observed by Jessop and Catcheside ( 1965$)$. This gene, which will be referred to as the rec-I gene, controls the frequency of allelic recombination. The dominant allele rec-I ${ }^{+}$reduces the frequency of allelic prototrophic recombinants in crosses between the same pair of alleles by a factor of more than ten without apparently affecting the frequency of non allelic recombination in adjacent segments. In the present study variations in frequency of allelic recombination are not much greater than twofold and scrutiny of the pedigrees of the stocks used indicates that all the crosses examined contained the dominant rec- $I^{+}$allele in at least one parent. Experiments designed to produce all the combinations of the rec- $I$ and rec-2 genetic differences are being made to determine the interactions if any of these genes.

Of considerable interest is the fact that the proximal and distal flanking marker differences associate independently with prototrophic allelic recombinants to give the four possible combinations. This seems to be true for other data (Catcheside, unpubl.). The present data are unique in that the distribution of flanking marker differences is homogeneous for crosses bearing the same rec-2 differences. In all other substantial bodies of data the distributions of the flanking marker differences, both proximal and distal, vary considerably. It is believed that the distribution of each of the flanking markers is subject to genetic control and it seems probable that the genetic controls of the distribution of the proximal marker act independently of those of the distal marker. Studies to identify the number and action of these controls have begun.

In Escherichia coli mutants have recently been demonstrated (Clark and Margulies, I965) which are highly sensitive to ultra violet light and also exhibit very low rates of recombination. Unlike the rec- $I$ and rec-2 genes of Neurospora low frequency of recombination in these mutants is a recessive character. These results seem to indicate that repair of radiation damage and genetic recombination are at least partly under common genetic controls. It may well be that the mechanisms of mutation and recombination involve a number of enzymes active in both processes. If this is so it follows that studies of recombination should be combined with studies of mutation. The relationships between the genetic control of recombinational and mutational events 
should provide invaluable information about the mechanism of both processes.

\section{SUMMARY}

Recombination was studied in crosses bearing four linked genetic differences, the middle two of which were allelic. Two distinct types of cross differed, by a factor of about two, in the frequency of recombinaof the two flanking markers. A single genetic difference controls the recombination frequency so that the dominant recombination gene reduces the frequency to about half that in crosses homozygous for the recessive one.

Prototrophic recombinants between the two centre allelic differences were selected and scored for their flanking marker constitution. The frequency of prototrophs bearing the less frequent proximal flanking marker changes by a factor of about $2 \cdot 3$, the lower frequency being determined by the dominant recombination allele.

The proximal and distal flanking markers associate independently with the prototrophic recombinants to give the four possible combinations.

Acknowledgments.-The author wishes to extend his sincere thanks to Professor D. G. Catcheside, F.R.S., for his encouragement and for much valuable discussion given throughout these investigations. Grateful thanks are also due to Miss Modra Eversons and Mr P. W. Macdonald for technical assistance.

\section{REFERENCES}

CATChEside, D. G. I960. Complementation among histidine mutants of Neurospora crassa. Proc. Roy. Soc. Lond., B153, I79-194.

CATCHESIDE, D. G., JESSOP, ADRIENNE P., AND SMITH, B. R. 1964. Genetic controls of allelic recombination in Neurospora. Nature, 202, $1242-1243$.

CLARK, A. J., AND MARGULIES, ANN DEE. 1965. Isolation and Characterisation of Recombination deficient mutants of Escherichia coli. Proc. Nat. Acad. Sci., Wash., $53,45^{\mathrm{I}-459}$.

hASTINGS, P. J., AND WHITEHOUSE, H. L. K. 1964. A polaron model of genetic recombination by the formation of hybrid DNA. Nature, 201, 1052-1054.

Holiday, R. 1962. Mutation and replication in Ustilago maydis. Genet. Res., 3, $472-486$.

HOLIDAY, R. I964. A mechanism for gene conversion in fungi. Genet. Res., 5, 282-303.

JESSOP, ADRIENNE P., AND CATCHESIDE, D. G. 1965. Interallelic recombination at the his-I locus in Neurospora crassa and its genetic control. Heredity, 20, 237-256.

sмiтн, в. R. 1962. Linkage data for group IV. Neurospora Neresletter, I, I6-1 7 .

SMITH, B. R. I 965 . Interallelic Recombination at the his-5 locus in Neurospora crassa. Heredity, 20, 257-276.

vogel, H. J. 1955. A convenient growth medium for Neurospora. Microbiol. Genet. Bull., 13, 42 .

WestergaARD, M., AND mitchell, H. K. 1947. Neurospora V. A synthetic medium favouring sexual reproduction. Amer. F. Bot., 34, 573-477.

WHITEHOUSE, H. L. K. I 963 . A theory of crossing over by means of hybrid deoxyribonucleic acid. Nature, 199, I034-1040.

Whitehouse, H. L. K., AND HASTINGS, P. J. I965. The analysis of genetic recombination on the polaron hybrid DNA model. Genet. Res., 6. 27-92. 